\title{
Trichomonas vaginalis and Tritrichomonas foetus: interaction with fibroblasts and muscle cells - new insights into parasite-mediated host cell cytotoxicity
}

\author{
Ricardo Chaves Vilela', Marlene Benchimol²/+ \\ 1Pós-graduação em Ciências Biológicas, Instituto de Biofísica Carlos Chagas Filho, \\ Universidade Federal do Rio de Janeiro, Rio de Janeiro, RJ, Brasil ${ }^{2}$ Universidade Santa Úrsula, Rio de Janeiro, RJ, Brasil
}

\begin{abstract}
Trichomonas vaginalis and Tritrichomonas foetus are parasitic, flagellated protists that inhabit the urogenital tract of humans and bovines, respectively. T. vaginalis causes the most prevalent non-viral sexually transmitted disease worldwide and has been associated with an increased risk for human immunodeficiency virus-1 infection in humans. Infections by $\mathrm{T}$. foetus cause significant losses to the beef industry worldwide due to infertility and spontaneous abortion in cows. Several studies have shown a close association between trichomonads and the epithelium of the urogenital tract. However, little is known concerning the interaction of trichomonads with cells from deeper tissues, such as fibroblasts and muscle cells. Published parasite-host cell interaction studies have reported contradictory results regarding the ability of $\mathrm{T}$. foetus and $\mathrm{T}$. vaginalis to interact with and damage cells of different tissues. In this study, parasite-host cell interactions were examined by culturing primary human fibroblasts obtained from abdominal biopsies performed during plastic surgeries with trichomonads. In addition, mouse 3 T3 fibroblasts, primary chick embryo myogenic cells and L6 muscle cells were also used as models of target cells. The parasite-host cell cultures were processed for scanning and transmission electron microscopy and were tested for cell viability and cell death. JC-1 staining, which measures mitochondrial membrane potential, was used to determine whether the parasites induced target cell damage. Terminal deoxynucleotidyltransferase-mediated dUTP nick end labelling staining was used as an indicator of chromatin damage. The colorimetric crystal violet assay was performed to analyse the cytotoxicity induced by the parasite. The results showed that $\mathrm{T}$. foetus and $\mathrm{T}$. vaginalis adhered to and were cytotoxic to both fibroblasts and muscle cells, indicating that trichomonas infection of the connective and muscle tissues is likely to occur; such infections could cause serious risks to the infected host.
\end{abstract}

Key words: Trichomonas vaginalis - Tritrichomonas foetus - cytotoxicity - parasite-host cell interaction

Trichomonas vaginalis, an extracellular, aerotolerant protist parasite, is the cause of trichomoniasis, the most prevalent non-viral sexually transmitted infection in the world (WHO 2001). Each year, 160-180 million people are infected with $T$. vaginalis worldwide (McClelland 2008). T. vaginalis infection is associated with serious adverse health consequences in women, including infertility (El-Shazly et al. 2001), atypical pelvic inflammatory disease (Moodley et al. 2002), pre-term delivery and delivery of low birth weight infants (Cotch et al. 1997) and predisposition to cervical neoplasia (Viikki et al. 2000). The effects of Tritrichomonas foetus infection in cows vary from mild vaginitis or cervicitis to endometritis, abortion and infertility (Parsonson et al. 1976, Anderson et al. 1996). Due to the considerable effects of $T$. vaginalis and $T$. foetus, more studies are necessary to address the unanswered questions surrounding the hostparasite interaction. Several studies have suggested that T. vaginalis and $T$. foetus closely associate with the epi-

Financial support: CNPq, FAPERJ, CAPES, PRONEX, AUSU +Corresponding author: marlenebenchimol@gmail.com Received 2 November 2011

Accepted 15 February 2012 thelial cells of the vagina. Although both parasites have been described in the upper parts of the reproductive tract, to date, there is no conclusive evidence to show that the final site of infection of these parasites is the host vagina (Aburel et al. 1957, Mancebo et al. 1995). Another important question is whether trichomonads are restricted to interactions with epithelial cells or are invasive and able to reach deeper tissues, such as the cells of the connective and muscle tissues. In the literature, some authors have claimed that $T$. vaginalis adheres to fibroblast monolayers, but the parasites were unable to provoke damage to this cell type (Alderete \& Garza 1985, Gilbert et al. 2000). In contrast, another group reported that $T$. vaginalis could invade and induce changes in deep vaginal tissues (Escario et al. 2010). Thus, it is necessary to investigate the nature and specificity of the behaviour of trichomonads using different cell types, such as human fibroblasts and muscle cells.

\section{SUBJECTS, MATERIALS AND METHODS}

Parasites - The JT strain of T. vaginalis was isolated at the University Hospital, Federal University of Rio de Janeiro (UFRJ), Brazil. An axenic culture has been maintained since the 1980s. The K strain of T. foetus was isolated by Dr H Guida (Embrapa, Rio de Janeiro, Brazil) from the urogenital tract of a bull. Parasites were cultivated in trypticase-yeast extract-maltose (TYM) medium 
(Diamond 1957) supplemented with $10 \%$ foetal bovine serum (FBS). Parasites were grown for $36-48 \mathrm{~h}$ at $37^{\circ} \mathrm{C}$, allowing them to enter the logarithmic growth phase.

Fibroblasts - Human fibroblasts were generously donated by Dr Radovan Borojevic (UFRJ). The cells were obtained from the mid-abdominal surgical incision; surgery was performed at the University Hospital, Department of Surgery, UFRJ. Informed consent was obtained from every patient and the study was conducted in agreement with the ethical guidelines of UFRJ. Biopsies were collected and brought to the laboratory in chilled Dulbecco's modified Eagle's medium (DMEM) (Sigma, St. Louis, MO) containing $10 \%$ fresh human serum and $60 \mu \mathrm{g} / \mathrm{mL}$ gentamicin (Schering, Rio de Janeiro, Brazil). Biopsy samples were cut into pieces of approximately $1 \mathrm{~mm}^{3}$, washed in fresh medium and plated at a density of $6-9$ pieces per $25-\mathrm{cm}^{2}$ flask. The results obtained in the mouse 3T3 fibroblast cell line, which was acquired from Cell Bank of Rio de Janeiro (BCRJ), were compared to those obtained in the human fibroblasts. 3T3 cells were cultured in DMEM containing 10\% FBS. The cells were maintained in DMEM supplemented with 4g/L HEPES and $10 \% \mathrm{FBS}$ at $37^{\circ} \mathrm{C}$ in a humidified incubator in the presence of $5 \% \mathrm{CO}_{2}$ and $95 \%$ air.

Muscle cells - Primary cultures of myogenic cells were prepared from the breast muscles of 11-day-old chick embryos as previously described (Mermelstein et al. 2005). The L6 skeletal muscle cell line was obtained from BCRJ and maintained in DMEM supplemented with $10 \%$ FBS. This lineage was originally obtained from muscle fragments of mouse thighs. The cells were cultured with $T$. vaginalis and $T$. foetus.

Co-incubation and attachment assays - For interaction analysis, host cell cultures were exposed to T. foetus $\mathrm{K}$ or $T$. vaginalis $\mathrm{JT}$ at a parasite to target cell ratio of 5:1 for $3-48 \mathrm{~h}$ at $37^{\circ} \mathrm{C}$. Prior to the addition of parasites, the cells were equilibrated in incubation medium containing two parts of complete DMEM ( $\mathrm{pH}$ 7.2) and one part of TYM Diamond's medium (W/D 2:1) at $37^{\circ} \mathrm{C}\left(5 \% \mathrm{CO}_{2}\right)$. Adhesion was confirmed by thorough washing of the cultured monolayers after exposure to parasites. In control experiments, the parasites were omitted. The progression of the interaction was followed by phase contrast microscopy using a Zeiss Axiophot II microscope (Oberkochen, Germany) within the first minute of addition of the parasites. Images were acquired using a Zeiss AxioCam MRc 5 (Germany).

Scanning electron microscopy (SEM) - After parasite interaction, coverslips were fixed with $2.5 \%$ glutaraldehyde in $0.1 \mathrm{M}$ sodium cacodylate buffer ( $\mathrm{pH}$ 7.2) for $2 \mathrm{~h}$. Afterwards, the coverslips were washed with PBS, post-fixed for $15 \mathrm{~min}$ in $1 \%$ osmium tetroxide $\left(\mathrm{OsO}_{4}\right)$, dehydrated in ethanol, critical point-dried with $\mathrm{CO}_{2}$ and sputter coated with gold-palladium. The samples were examined with a JEOL 5800 scanning electron microscope (Akishima, Tokyo, Japan).

Transmission electron microscopy (TEM) - Cells were fixed in $2.5 \%(\mathrm{v} / \mathrm{v})$ glutaraldehyde, post-fixed for
$15 \mathrm{~min}$ in $1 \% \mathrm{OsO}_{4}$, dehydrated in acetone and embedded in Epon. Ultra-thin sections were observed with a JEOL 1210 transmission electron microscope.

Mitochondrial membrane potential - The JC-1 fluorescent dye (Molecular Probes, USA) was used to quantitatively assess changes in mitochondrial membrane potential following trichomonas infection. After parasite-cell interaction, the remaining host cells were incubated with JC-1 $(10 \mu \mathrm{g} / \mathrm{mL}$ in dimethyl sulfoxide at $37^{\circ} \mathrm{C}$ for $45 \mathrm{~min}$. JC-1 selectively enters mitochondria, where it can be used to measure the magnitude of the mitochondrial membrane potential based on the detection of either green fluorescent monomers (depolarised) or red fluorescent aggregates (polarised). The ratio of red to green JC-1 fluorescence intensity serves as an index of the mitochondrial membrane potential; a higher ratio indicates a greater membrane potential. JC-1 fluorescence was observed using a Zeiss Axiophot II fluorescent microscope (Zeiss, Germany) equipped with a phase-contrast $40 \mathrm{X} / 0.75$ na objective. JC-1 green fluorescence was observed with a standard fluorescein filter set (excitation BP 450-490 nm, emission LP $520 \mathrm{~nm}$ ) and JC-1 red fluorescence was observed with a standard rhodamine filter set (excitation BP 534-558 nm, emission LP $590 \mathrm{~nm}$ ). Images were randomly acquired with a high-resolution digital camera (AxioCam MRc5, Zeiss, Germany). All images were acquired using the same exposure time, brightness and contrast. The ratio of red to green fluorescence intensity was determined using ImageJ (version 1.43) software (National Institutes of Health, USA) and is expressed in arbitrary units. The data are expressed relative to the ratio of red to green fluorescence intensity in control cells, which was given a value of $100 \%$. Fifteen digital images per replicate were analysed. The results are presented as the average of three independent experiments.

Cytotoxicity assays - For cytotoxicity analysis, the host cells were grown to confluence in 24-well plates (approximately $2 \times 10^{5}$ cells) and exposed to $T$. foetus $\mathrm{K}$ or T. vaginalis $\mathrm{JT}$ at a parasite to target cell ratio of 5:1 for $3-48 \mathrm{~h}$ at $37^{\circ} \mathrm{C}$. The cells were equilibrated in incubation medium containing two parts of complete DMEM (pH 7.2) and one part of TYM Diamond's medium (W/D 2:1) for $15 \mathrm{~min}$ at $37^{\circ} \mathrm{C}\left(5 \% \mathrm{CO}_{2}\right)$ prior to the addition of parasites. In control experiments, the parasites were omitted. At the end of the incubation periods, the wells were gently washed twice (being careful to avoid the disruption of the remaining monolayer) with warm PBS and the cells that were still adherent were fixed with $2 \%(\mathrm{wt} / \mathrm{vol})$ paraformaldehyde $(\mathrm{PF})$ in phosphate buffer for $15 \mathrm{~min}$ at room temperature (RT). The wells were washed with PBS and the remaining cells were stained with $0.13 \%$ crystal violet dissolved in a 5:2 (vol/vol) ethanol-PF solution. The stained product was subsequently washed twice with distilled water and air dried. Finally, the stained cells were solubilised in $1 \%(\mathrm{wt} / \mathrm{vol})$ sodium dodecyl sulfate in $50 \%(\mathrm{vol} / \mathrm{vol})$ ethanol and the intensity of staining was read in a spectrophotometer at a wavelength of $570 \mathrm{~nm}$. Each experiment was performed in 
triplicate and the data are presented as mean values. $\mathrm{Cy}$ totoxicity was calculated as $1-(E / C)$ : all measurements of experimental $(E)$ samples $\left(A_{570}\right)$ were indexed to those of control $(C)$ samples $(E / C)$, which showed no loss of cells from the well and subtracted from 1.0.

Terminal deoxynucleotidyltransferase-mediated dUTP nick end labelling (TUNEL) - The TUNEL assay was performed using the In Situ Cell Death Detection Kit (Roche Diagnostics, Meylan, France). Cells were adhered to coverslips, fixed at RT in $2 \% \mathrm{PF}(\mathrm{v} / \mathrm{v})$ in $0.1 \mathrm{M}$ phosphate buffer ( $\mathrm{pH}$ 7.2), permeabilized with $3 \%$ Nonidet-40 (St Louis, USA) for $40 \mathrm{~min}$ and quenched in $50 \mathrm{mM}$ ammonium chloride containing $3 \%(\mathrm{w} / \mathrm{v})$ bovine serum albumin in PBS. The labelling and signal conversion processes were carried out according to the manufacturer's instructions. The positive control slides were treated with DNase (Sigma, USA) for $10 \mathrm{~min}$ at RT. The negative control slides were not treated with DNase. The slides were observed with a fluorescence microscope (Axiophot II, Zeiss, Germany) and the images were acquired using a chilled AxioCam MRc5 camera (Zeiss, Germany).

\section{RESULTS}

Trichomonad infection of human and mouse fibroblasts - To investigate the effects of $T$. vaginalis and $T$. foetus on connective tissue cells, primary human and mouse fibroblasts were cultured with the parasites. The uninfected human fibroblasts used as a control formed a non-confluent monolayer (Fig. 1A) with cells presenting several projections (Fig. 1B). The parasites were added to the fibroblast culture and analysed by SEM after 3-48 h of interaction. Both $T$. vaginalis (Fig. 1C) and $T$. foetus (Fig. 1D) attached to the fibroblast monolayer. To analyse whether the attached parasites induced damage or cell death in the fibroblasts in the monolayer, the JC-1 staining test was used to investigate the integrity or loss of mitochondrial membrane potential. As expected, the uninfected fibroblasts exhibited healthy mitochondria with JC-1 red fluorescence (Fig. 2B), whereas infected human fibroblasts co-incubated with $T$. vaginalis (Fig. 2F) or T. foetus (Fig. 2I) showed a loss of mitochondrial membrane potential. After $12 \mathrm{~h}$ of interaction, the infected cells did not exhibit any red fluorescence (Fig. 2E, $\mathrm{H}$ ), thus confirming the complete loss of mitochondrial potential. For comparison with human fibroblasts, host cell-parasite interactions were performed with mouse 3T3 fibroblasts. Non-infected (control) 3T3 cells exhibited a sub-confluent appearance and were extended and flat (Fig. 3A). In addition, small microvilli were occasionally observed (Fig. 3B, arrows). After the addition of the parasites, T. vaginalis attached to 3T3 fibroblasts, even when the parasite was in the process of cell division (Fig. 3C). The addition of T. foetus to 3T3 cells led to host cell retraction (Fig. 3D) and membrane blebbing (Fig. 3D, asterisk), which are morphological changes indicative of apoptosis. When the JC-1 test was performed, the mitochondria in control cells exhibited red fluorescence (Fig.
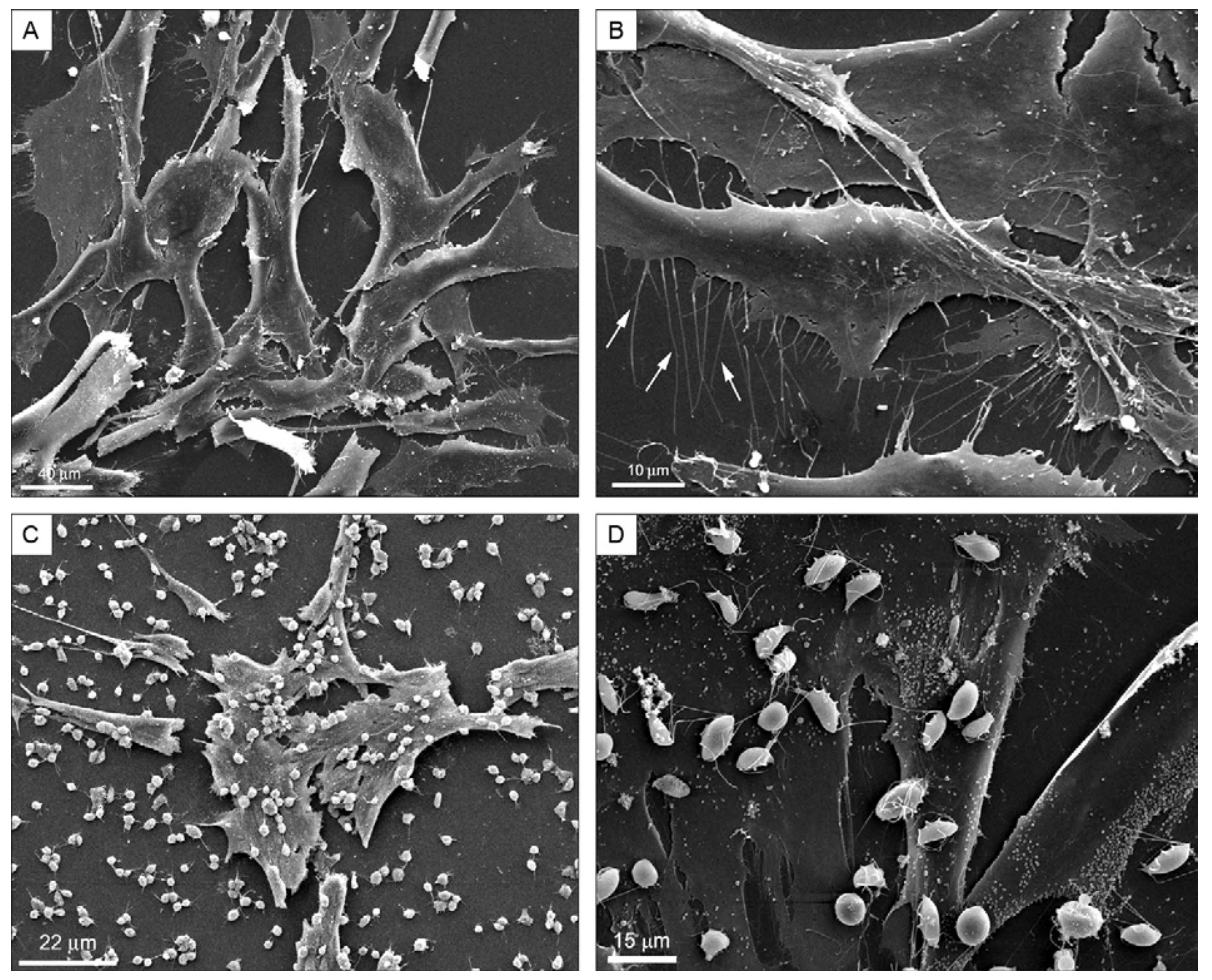

Fig. 1: scanning electron microscopy of the interaction of Trichomonas vaginalis and Tritrichomonas foetus with human fibroblasts. In the controls (A, B) parasites were not added. A: a non-confluent monolayer of human fibroblasts. Note a high number of fillopodia emitted by fibroblasts (B, arrows); C, D: display interaction of parasites T. vaginalis (C) and T. foetus (D) adhered to human fibroblasts within $3 \mathrm{~h}$ of interaction. 
4B), whereas those in infected $3 \mathrm{~T} 3$ fibroblasts showed green fluorescence (Fig. 4F, I), thus confirming the loss of mitochondrial membrane potential. The endonuclease activity was also evaluated using the TUNEL apoptosis detection assay. The DNase-treated positive control showed several positively labelled nuclei (Fig. 5A, B).
In contrast, no fluorescence was observed in the negative control sample that was not incubated with parasites (Fig. 5C, D). Apoptotic nuclei were observed $12 \mathrm{~h}$ after infection with T. vaginalis (Fig. 5D, E). Interestingly, the 3T3 fibroblasts did not exhibit labelling when $T$. foetus was used for the interaction experiments (Fig. 5G, H).
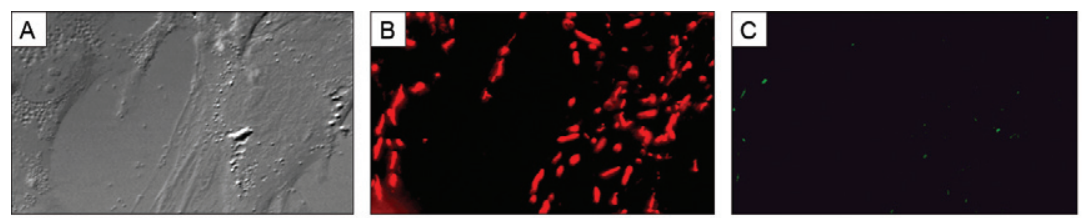

$$
\begin{gathered}
\text { Human } \\
\text { fibroblasts } \\
\text { control }
\end{gathered}
$$
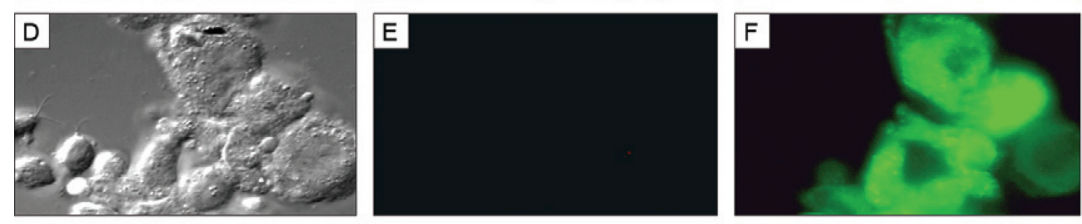
Human
fibroblasts

$+$

T. vaginalis $\mathrm{JT}$

$12 \mathrm{~h}$
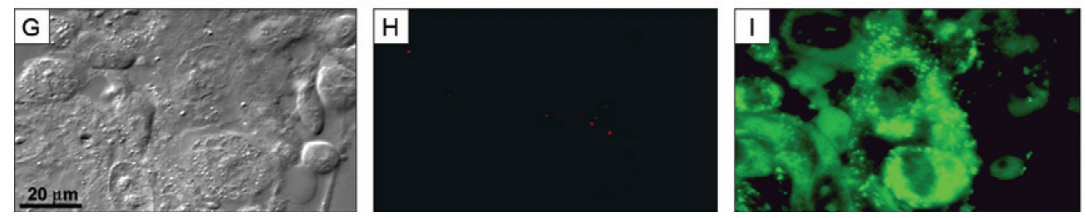

Human

fibroblasts

$+$

T. foetus $\mathrm{K}$

$12 \mathrm{~h}$

Fig. 2: mitochondrial viability test using JC-1 performed after interaction of human fibroblasts with trichomonads. A-C: control, human fibroblasts without parasites; D-F: Trichomonas vaginalis in interaction with human fibroblasts; G-I: Tritrichomonas foetus after $12 \mathrm{~h}$ of interaction with human fibroblasts. Note that when no parasites are added, almost all mitochondria fluoresces in red, indicating normal mitochondria membrane potential (B), whereas after co-incubation with $T$. vaginalis or T. foetus this potential is lost because mitochondria present green fluorescence (F, I); A, D, G: differential interferential contrast; B, E, H: red filter; C, F, I: green filter.
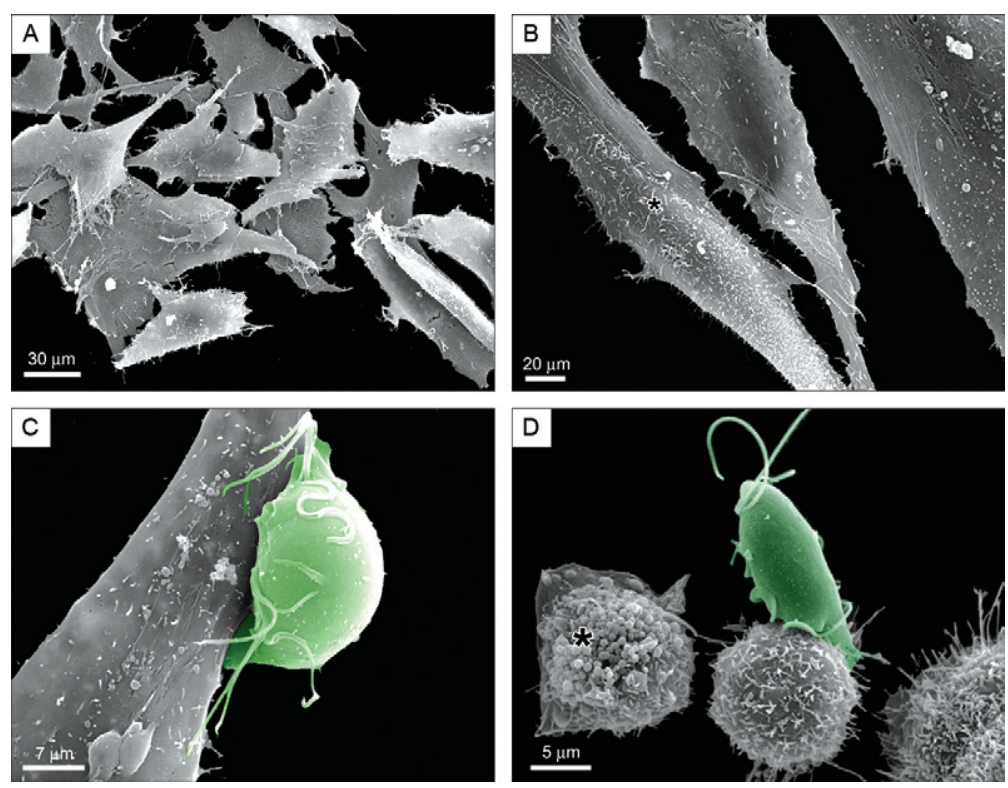

Fig. 3: scanning electron microscopy of the interaction between Trichomonas vaginalis and Tritrichomonas foetus with mouse fibroblasts. A, B: controls, the parasites were not added; A: a typical non-confluent fibroblast monolayer formation; B: in higher magnification small microvilli formation can be seen (asterisk); C, D: interaction of parasites T. vaginalis (C) and T. foetus (D) with murine fibroblasts. After 12 $\mathrm{h}$ of interaction both parasites are tightly adhered to the fibroblasts which already present signs of damage. In $\mathrm{C}, T$. vaginalis is attached even when the parasite is in process of division. Note in D that $T$. foetus preferentially adheres by its posterior region. Host cells present cell retraction and membrane blebbing (D, asterisk). 
Trichomonad interaction with skeletal muscle cells To investigate the ability of trichomonads to infect muscle cells, two muscle cell cultures were used as host cells: (i) a primary culture of myoblasts and (ii) the L6 muscle cell line. SEM analysis showed that control myoblasts were confluent, elongated and fusiform cells that presented several fibres (Fig. 6A, B). During the interaction assays, both T. vaginalis (Fig. 6C, D) and T. foetus (Fig. 6E, F) were found attached to the host cells; after $6 \mathrm{~h}$ of interaction, retraction of the host cell was clearly observed. TEM images confirmed that both T. vaginalis (Fig. 7A, B) and T. foetus (Fig. 7C, D) attached to muscle cells and exhibited close membrane-membrane contact. When the primary culture of muscle cells was used, similar findings were observed (data not shown). In addition, cells that presented morphological signs of apoptosis were detected (Fig. 7B): condensed chromatin and intense vacuolisation were frequently observed. T. foetus was able to adhere simultaneously to three or more cells (Fig. 7C) and later on to endocytic activity, pulling in the muscle cell (Fig. 7D). To confirm whether trichomonad infection induced host cell damage or cell death in muscle cells, both the muscle primary culture and the L6 muscle cells were tested using JC-1 staining to measure mitochondrial membrane potential after incubation with trichomonas. Red fluorescence was observed in the mitochondria of cells to which no parasites were added (Fig. 8B), demonstrating normal mitochondrial membrane potential. In contrast, mitochondria exhibited green fluorescence $6 \mathrm{~h}$ after muscle cells were combined with T. vaginalis (Fig. 8 F) or T. foetus (Fig. 8I), indicating that these parasites induced a loss of mitochondrial membrane potential. Both types of muscle cells yielded similar results.

Parasite-mediated cytotoxicity in host tissues - The crystal violet assay was used to quantitatively assess the trichomonad-induced damage to the host cells tested here (Fig. 9). When combined with 3T3 fibroblasts, T. vagi- nalis disrupted $60 \%$ of the monolayer in $48 \mathrm{~h}$, whereas T. foetus did not cause significant levels of cytotoxicity. After $6 \mathrm{~h}$ of interaction with human fibroblasts, $T$. vaginalis and T. foetus disrupted $90 \%$ and $60 \%$ of the
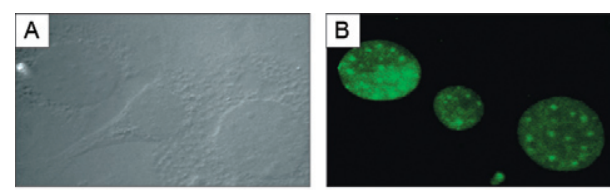

Positive control 3T3 fibroblasts. $+$ DNase
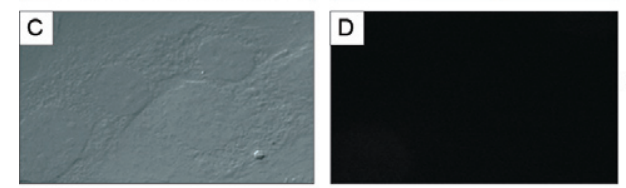

3T3 fibroblasts control
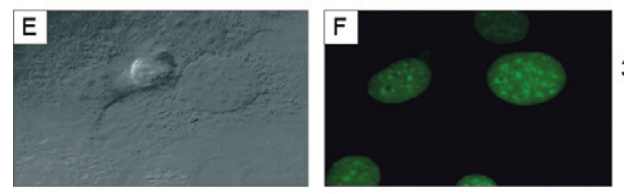

$3 T 3$ fibroblasts

T. vaginalis $\mathrm{JT}$
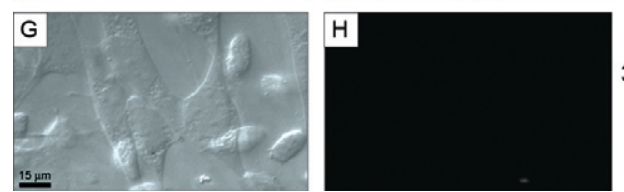

3 T 3 fibroblasts $+$

T. foetus $\mathrm{K}$

Fig. 5: murine $3 \mathrm{~T} 3$ fibroblasts before (A-D) and after $12 \mathrm{~h}$ of interaction with Trichomonas vaginalis (E, F) or Tritrichomonas foetus $(\mathrm{G}, \mathrm{H})$. A, C, E, G: differential interferential contrast; B, D, F, H: terminal deoxynucleotidyltransferase-mediated dUTP nick end labelling (TUNEL) test observed by fluorescence microscopy; A, B: control. 3T3 fibroblasts, no parasites added, after DNase I treatment. The nuclei present positive labelling; C, D: control. Murine fibroblasts without parasite interaction followed by TUNEL test. No labelling is observed, indicating that chromatin is in good conditions; E, F: 3T3 cells co-incubated with T. vaginalis for $12 \mathrm{~h}$. The nuclei present intense positive labelling indicating damage to DNA of the host cells; G, H: T. foetus incubated for $12 \mathrm{~h}$ with $3 \mathrm{~T} 3$ mouse fibroblasts as target cells. No TUNEL labelling is observed.
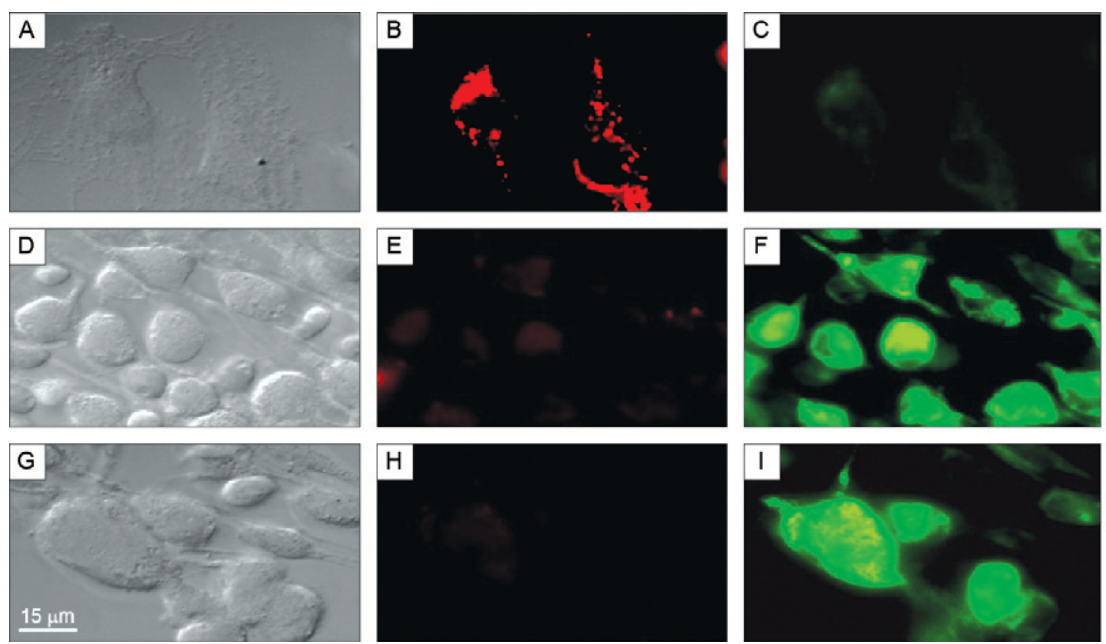

3T3 fibroblasts

control

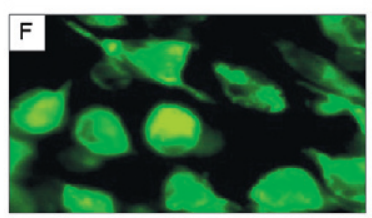

$3 T 3$ fibroblasts

T. vaginalis $+\mathrm{JT}$

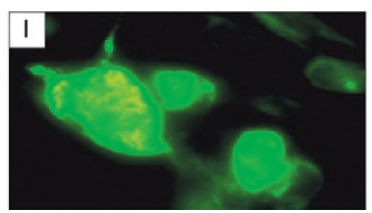

$3 \mathrm{~T} 3$ fibroblasts

$+$

T. foetus $+\mathrm{K}$

Fig. 4: mitochondrial viability test using JC-1 before (A-C) and after (G-I) the interaction of 3T3 mouse fibroblasts with trichomonads. A-C: control, 3 T3 fibroblasts without added parasites. Note that mitochondria fluoresce in red indicating membrane potential (B); D-F: Trichomonas vaginalis strain JT in interaction with $3 \mathrm{~T} 3$ mice fibroblasts. Notice that a light red fluorescence is seen (E), whereas almost all mitochondria fluoresce in green (F) indicating loss of mitochondria membrane potential; G-I: Tritrichomonas foetus after $12 \mathrm{~h}$ of interaction. Nearly all cells present green fluorescence (I); A, D, G: differential interferential contrast; B, E, H: red filter; C, F, I: green filter. 
cell monolayer, respectively (Fig. 9B). When the parasites were added to both cultures of muscle cells, it was observed that both parasites were efficient to at disrupting host cell monolayers, although $T$. vaginalis disrupted $80 \%$ in $10 \mathrm{~h}$, whereas T. foetus performed dispersed it $50 \%$ in the same time (Fig. 9C).

\section{DISCUSSION}

Human and bovine trichomoniasis can lead to symptoms such as colpitis macularis (Heine \& McGregor 1993), infertility (Felleisen 1999) and abortion (BonDurant 1997). Trichomonads are always described as extracellular, non-invasive parasites. However, small wounds are frequently found in women and cows infected by trichomonads, but no plausible explanation has been proposed. Because the consequences of trichomonas infections are not fully understood, these wounds could be the result of the damage exerted by these parasites on deeper tissues of the urogenital region, such as connective tissue and muscle.

The vaginal epithelial layer is thick and stratified and trichomonads are known as non-invasive vaginal epithelial parasites. However, published reports have described trichomonas-infected patients with parasites present in the upper genital tract (Aburel et al. 1957, Skirrow \& BonDurant 1990), including the uterus and oviducts. The presence of a single epithelial layer in these organs could facilitate the parasite's penetration into the organ.

In the present work, the capacity of $T$. vaginalis and T. foetus to attach to and infect host cells distinct from epithelial cells, such as those localised in the deeper tissues of the body (e.g., connective and muscle tissues) was investigated. For these studies, we used both cell lines and primary cultures.

Previous in vitro studies have addressed the interaction of T. vaginalis with human fibroblasts (Alderete \& Garza 1985, Gilbert et al. 2000). The authors of these studies concluded that the parasites were able to attach to the cells, but did not induce cytotoxicity (Alderete \& Garza 1985, Gilbert et al. 2000). In addition, recent work in which mice were used as a host model for T. vaginalis infection demonstrated that trichomonas causes alterations in the connective tissue (Escario et al. 2010). However, no studies of trichomonas-host cell interactions have been performed in muscle cells and the interaction of the parasite T. foetus with fibroblasts has not previously been examined. Thus, we aimed to clarify some of the still controversial and obscure aspects of trichomonas infection. To this end, we studied two types of fibroblasts ( 1 from a human source and the other a lineage from mice) as well as a primary culture obtained directly from muscle. In addition, we examined parasite-host cell interactions using two trichomonas species, $T$. vaginalis and T. foetus. The infection process was analysed by SEM dramatic monolayer retraction was the first consequence of the attachment of the parasite to the target cells. Further cytotoxicity analyses using the crystal violet test as a measure of cytotoxicity were performed. This test was similarly employed by Alderete and Garza (1985) in parasite-host cell interaction studies using fibroblasts as
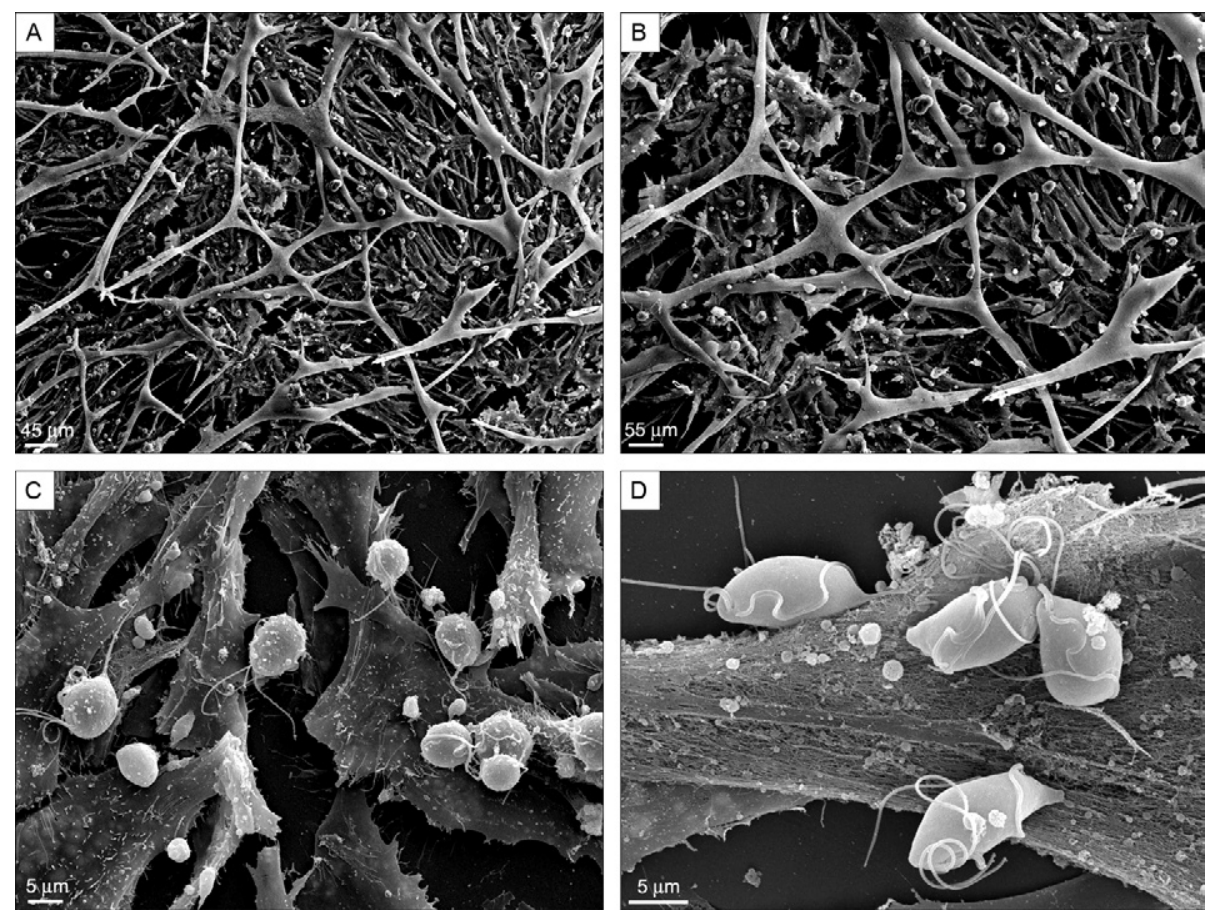

Fig. 6: scanning electron microscopy analysis of skeletal muscle cells monolayer before (A, B) and after (C, D) parasites interaction for $6 \mathrm{~h}$. In the controls (A, B), no parasites were added and the muscle cells exhibit confluent and elongated fibres. Trichomonas vaginalis (C, D) and Tritrichomonas foetus (E, F) were added to the confluent muscle cultures and were able to attach and retract the target cells after $6 \mathrm{~h}$ of interaction. Notice in C-F the open spaces in the muscle cells culture after parasites addition. 
target cells. In our hands, the crystal violet test demonstrated that trichomonas caused a high degree of cytotoxicity. This finding was corroborated by our morphological data. The divergence of our findings from previous work (Alderete \& Garza 1985, Gilbert et al. 2000) might be explained by differences in the trichomonad strains used, as different trichomonad strains induce distinct effects on cytotoxicity (Jesus et al. 2004).
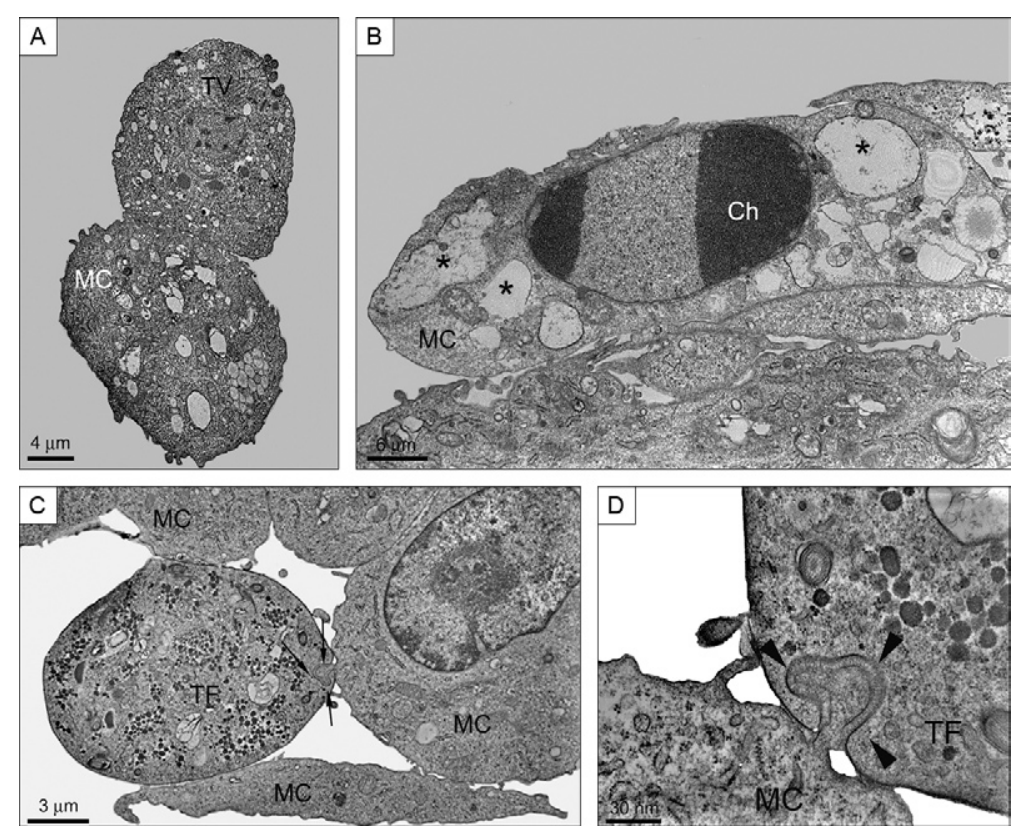

Fig. 7: transmission electron microscopy of interaction of Trichomonas vaginalis (TV) with muscle cells (A, B) and Tritrichomonas foetus (TF) (C, D) for $6 \mathrm{~h}$. A: an attachment between T. vaginalis and one muscle cell is clearly seen. Notice as the muscle cell is retracted and have lost its elongated and fusiform shape as seen in the control cultures; B: two muscle cells are seen and one of them presents morphological characteristics of apoptosis, such as condensed chromatin (ch) and intense vacuolization (asterisks); C, D: interaction of T. foetus with muscle cells; C: note an intimate and simultaneous contact exerted by one parasite with three target cells; D: detail of an endocytic process where $T$. foetus is pulling a muscle cell portion (arrowheads); MC: muscle cell.
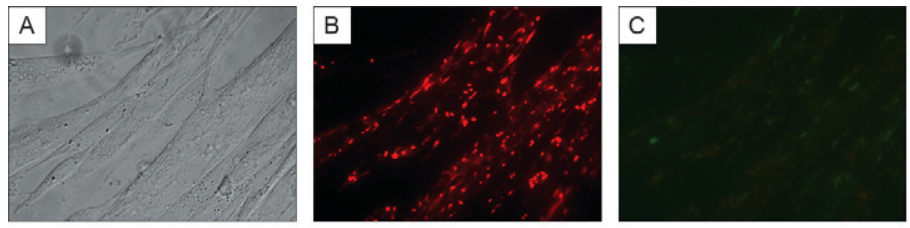

Muscle cells control
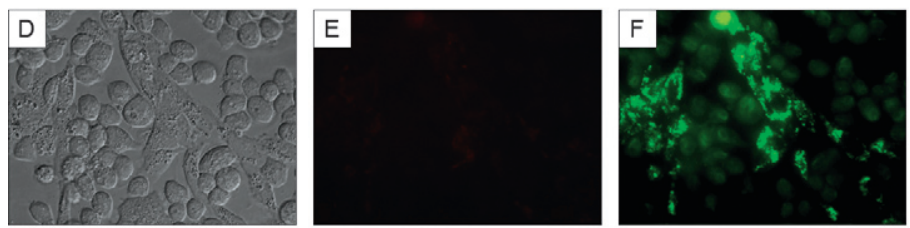

Muscle cells

$+$

T. vaginalis $\mathrm{JT}$
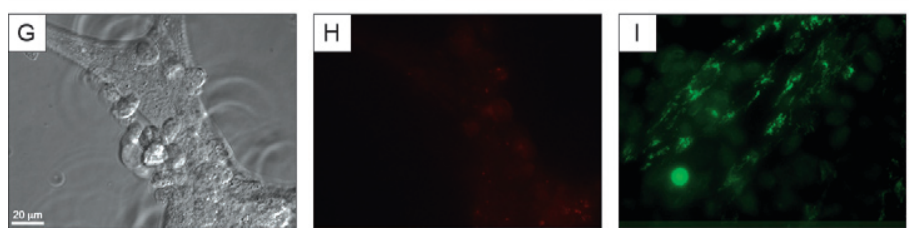

Muscle cells $+$

T. foetus $\mathrm{K}$

Fig. 8: mitochondrial viability test using JC-1 after the interaction of primary culture of muscle cells with trichomonads. A-C: control, muscle cells without parasites; D-F: Trichomonas vaginalis in interaction with muscle cells, 6 h; G-I; Tritrichomonas foetus after 6 h of interaction. Note that when no parasites are added, almost all mitochondria fluoresces in red, indicative of normal mitochondria membrane potential (B), whereas after co-incubation with T. vaginalis or T. foetus this potential was lost (mitochondria are in green fluorescence colour) (F, I). A, D, G: differential interferential contrast; B, E, H: red filter; C, F, I: green filter. 


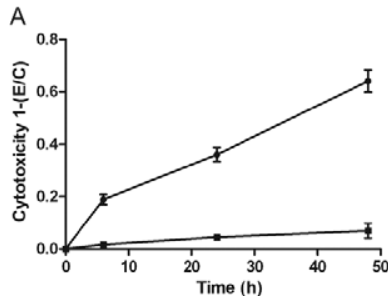

B
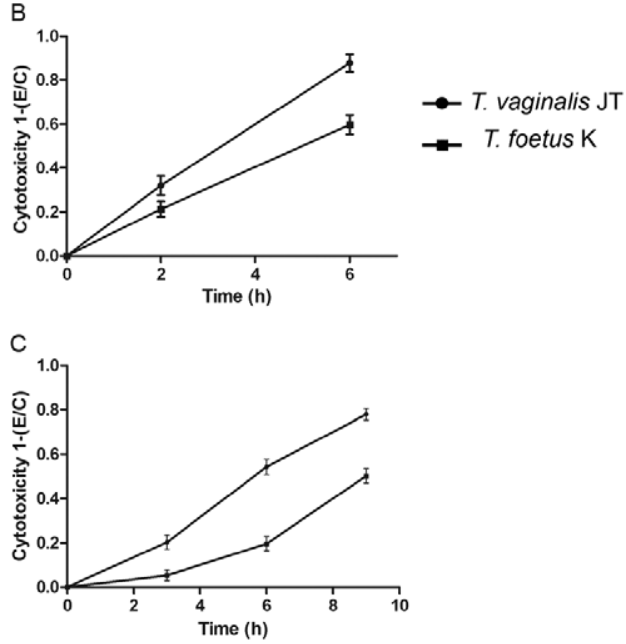

Fig. 9: crystal-violet test. The graphics show the results of interaction of Trichomonas vaginalis or Tritrichomonas foetus with 3T3 mouse fibroblasts (A), human fibroblasts (B) and muscle cells (C). Both parasites were efficient to damage host cells and disrupted host cell monolayers, although $T$. vaginalis was more efficient because disrupted $80 \%$ in $10 \mathrm{~h}$, whereas $T$. foetus performed $50 \%$ in the same time (C). Cytotoxicity was determined after staining the remaining fixed cells in microtitre plate wells and subtracting the ratio of absorbance at $570 \mathrm{~nm}$ of the experimental (E) to control (C) samples from 1.

Although T. foetus was unable to disrupt the $3 \mathrm{~T} 3$ fibroblast monolayer efficiently, the parasites were able to adhere and induce alterations to the mitochondrial membrane potential, as demonstrated by JC-1 staining. When the 3T3 fibroblasts were used as target cells for interaction with $T$. foetus, no positive labelling was demonstrated by TUNEL assay. This result could be due to either a low degree of cytotoxicity of $T$. foetus in this cell line or a distinct behaviour of T. foetus when compared to $T$. vaginalis. Importantly, both $T$. vaginalis and T. foetus were capable of disrupting both types of muscle cell monolayers that were used in this study.

Considering the results presented here and taking into account the single epithelial monolayer present in the uterus and oviducts, we suggest that trichomonads could destroy the single epithelial layer in these organs and invade the deeper tissues, inducing damage to fibroblasts and muscle cells. Further in vivo studies are important to confirm our observations and potentially change the current thinking about trichomonas infection.

\section{ACKNOWLEDGEMENTS}

To Dr Claudia Mermelstein, for the muscle cells donation, and to Dr Radovan Borojevic and Ana Paula Dantas, for providing the human fibroblasts.

\section{REFERENCES}

Aburel E, Oltet, Niculescu, Sufrin 1957. Presence of Trichomonas vaginalis in the upper genital tract. CR Soc Fr Gyncol 27: 409-413.

Alderete JF, Garza JE 1985. Specific nature of Trichomonas vaginalis parasitism of host cell surfaces. Infect Immun 50: 701-708.

Anderson ML, Bondurand RH, Corbeil RR, Corbeil LB 1996. Immune and inflammatory responses to reproductive tract infection with Tritrichomonas foetus in immunized and control heifers. J Parasitol 82: 594-600.

BonDurant RH 1997. Pathogenesis, diagnosis and management of trichomoniasis in cattle. Vet Clin North Am Food Anim Pract 13: 345-361.

Cotch MF, Pastorek JG, Nugent RP, Hillier SL, Gibbs RS, Martin DH 1997. Trichomonas vaginalis associated with low birth weight and preterm delivery. The vaginal infections and prematurity study group. Sex Transm Dis 24: 353-360.

Diamond LS 1957. The establishment of various Trichomonas of animals and man in axenic cultures. J Parasitol 43: 488-490.

El-Shazly AM, El-Naggar HM, Soliman M, El-Negeri M, El-Nemr HE, Handousa AE, Morsy TA 2001. A study on Trichomonas vaginalis and female infertility. J Egypt Soc Parasitol 31: 545-553.

Escario A, Gómez Barrio A, Simons Diez B, Escario JA 2010. Immunohistochemical study of the vaginal inflammatory response in experimental trichomoniasis. Acta Trop 114: 22-30.

Felleisen RSJ 1999. Host-parasite interaction in bovine infection with Tritrichomonas foetus. Microbes Infect 1: 807-816.

Gilbert RO, Elia GD, Beach H, Klaessig S, Singh BN 2000. Cytopathogenic effect of Trichomonas vaginalis on human vaginal epithelial cells cultured in vitro. Infect Immun 68: 4200-4206.

Heine P, McGregor JA 1993. Trichomonas vaginalis: a reemerging pathogen. Clin Obstet Gynecol 36: 137-144.

Jesus JB, Vannier-Santos MA, Britto C, Godefroy P, Silva-Filho FC, Pinheiro AA, Rocha-Azevedo B, Lopes AH, Meyer-Fernandes JR 2004. Trichomonas vaginalis virulence against epithelial cells and morphological variability: the comparison between a wellestablished strain and a fresh isolate. Parasitol Res 5: 369-377.

Mancebo OA, Russo AM, Carabajal LL, Monzon CM 1995. Persistence of Tritrichomonas foetus in naturally infected cows and heifers in Argentina. Vet Parasitol 59: 7-11.

McClelland RS 2008. Trichomonas vaginalis infection: can we afford to do nothing? J Infect Dis 197: 487-489.

Mermelstein CS, Portilho DM, Medeiros RB, Matos AR, EinickerLamas M, Tortelote GG, Vieyra A, Costa ML 2005. Cholesterol depletion by methyl- $\beta$-cyclodextrin enhances myoblast fusion and induces the formation of myotubes with disorganized nuclei. Cell Tissue Res 319: 289-297.

Moodley P, Wilkinson D, Connolly C, Moodley J, Sturm AW 2002. Trichomonas vaginalis is associated with pelvic inflammatory disease in women infected with human immunodeficiency virus. Clin Infect Dis 34: 519-522.

Parsonson IM, Clark BL, Dufty JH 1976. Early pathogenesis and pathology of Tritrichomonas foetus infection in virgin heifers. J Comp Pathol 86: 59-66.

Skirrow SZ, BonDurant RH 1990. Induced Tritrichomonas foetus infection in beef heifers. J Am Vet Med Assoc 196: 885-889.

Viikki M, Pukkala E, Nieminen P, Hakama M 2000. Gynaecological infections as risk determinants of subsequent cervical neoplasia. Acta Oncol 39: 71-75.

WHO - World Health Organization 2001. Global prevalence and incidence of selected curable sexually transmitted infections. Overview and estimates, WHO, Geneva, $42 \mathrm{pp}$. 